\title{
Risk for Hepatitis B Virus Reactivation in Patients with Psoriasis Treated with Biological Agents: A Systematic Review and Meta-Analysis
}

\author{
Xinyu Wang · Ming Zhang · Yu Chen · Yirong Liu • Yan Yu • \\ Xiaojie Huang · Yanqing Gao (D)
}

Received: November 26, 2021 / Accepted: January 10, 2022 / Published online: January 29, 2022

(C) The Author(s) 2022

\begin{abstract}
Introduction: Notwithstanding their numerous advantages, biological treatments have many limitations when treating patients with psoriasis ( $\mathrm{PsO})$ and hepatitis B (HB). Clinicians need to pay careful attention to the issue of hepatitis $B$ virus (HBV) reactivation.
\end{abstract}

Xinyu Wang and Ming Zhang contributed equally to this work and share first authorship.

Supplementary Information The online version contains supplementary material available at https:// doi.org/10.1007/s13555-022-00682-5.

X. Wang · M. Zhang · Y. Yu · Y. Gao ( $\varangle)$ Department of Dermatology, Beijing Youan Hospital, Capital Medical University, Beijing, China e-mail: gaoyanqing@ccmu.edu.cn

Y. Chen

Fourth Department of Liver Disease (Difficult and Complicated Liver Diseases and Artificial Liver Center), Beijing Youan Hospital, Capital Medical University, Beijing, China

Y. Liu

Outpatient Department, Beijing Youan Hospital, Capital Medical University, Beijing, China

X. Huang $(\bowtie)$

Center for Infectious Diseases, Beijing Youan Hospital, Capital Medical University, Beijing, China e-mail: huangxiaojie78@ccmu.edu.cn

Y. Gao

Candidate Branch of National Clinical Research

Center for Skin Diseases, Beijing, China
Methods: In accordance with the PRISMA guidelines, we systematically searched Pubmed, Scopus, Embase, Cochrane Library, and Web of Science databases for observational studies on the topic of HBV reactivation among patients with $\mathrm{PsO}$ and $\mathrm{HB}$ treated with biologics. The random-effects model was used to pool the reactivation rate by the Freeman-Tukey double arcsine transformation method. We selected Fisher's exact test to compare multiple rates. To determine the sources of heterogeneity, sensitivity analysis and meta-regression were performed.

Results: Ten studies with a total of 238 subjects that met the inclusion criteria were included. The pooled reactivation rate was $1.8 \%$ [95\% confidence interval (CI) $0.0-5.6 \%$ ] in patients with $\mathrm{PsO}$ and $\mathrm{HB}$. Among them, the viral reactivation rates of HBsAg-positive and HBsAgnegative patients were $4.1 \% \quad(95 \% \mathrm{CI}$ $0.0-17.9 \%)$ and $0.2 \%$ (95\% CI $0.0-2.8 \%)$. The difference between HBsAg-positive and HBsAgnegative patients was statistically significant $(p=0.002)$. The viral reactivation rate of individuals who needed antiviral prophylaxis but did not receive it was $26.6 \% \quad(95 \% \mathrm{CI}$ $5.8-53.5 \%)$, while it decreased to $0.0 \%$ (95\% CI $0.0-6.6 \%)$ after accepting antiviral treatment. The two-sided Fisher's test exact values between different durations of biological therapy showed no statistical significance $(p=0.104)$.

Conclusions: Without antiviral prophylaxis, HBsAg-positive patients with psoriasis are at 
high risk of virus reactivation when treated with biological agents. Early and sufficient antiviral prophylaxis will effectively reduce the risk of HBV reactivation and serious complications in HBsAg-positive patients. Prolonging the duration of biological treatment will not increase the risk of reactivation.

Keywords: Hepatitis B virus; Hepatitis B; Psoriasis; Psoriatic arthritis; Biological treatment; Biological agent; Virus reactivation

\section{Key Summary Points}

Current studies on the hepatitis B virus reactivation rate of patients with psoriasis treated with biological agents contain only a small number of cases.

This study containing 238 cases quantified the risk of viral reactivation in this at-risk population and evaluated the related risk factors.

The pooled result showed that the viral reactivation rate of $\mathrm{HBsAg}$-positive patients with psoriasis was $4.1 \%$ (95\% confidence interval, $0.0-17.9 \%, I^{2}$ statistic $=0.00 \%)$.

Without antiviral prophylaxis, HBsAgpositive patients with psoriasis are at high risk of virus reactivation when treated with biological agents. Early and sufficient antiviral prophylaxis will effectively reduce the risk of $\mathrm{HBV}$ reactivation and serious complications in $\mathrm{HBsAg}$-positive patients. Prolonging the duration of biological treatment will not increase the risk of reactivation.

\section{INTRODUCTION}

Since their inception, biological agents have been widely applied to various kinds of autoimmune diseases. The characteristics of excellent convenience, considerable efficacy, and satisfactory compliance have made them the first choice [1] after the failure of or intolerance to traditional treatment for many chronic illnesses, such as ankylosing spondylitis [2], inflammatory bowel disease [3], rheumatoid arthritis [4], and psoriasis (PsO) [5].

However, from the perspective of safety, the clinical applications of biologics still have numerous precautions and contraindications [6]. For instance, patients with malignant tumors or various active infections should be strictly prohibited from using biological products. The anti-inflammatory role of biological agents in treating moderate, severe, and refractory psoriasis by acting on cytokines or receptors may also lead to HBV replication and reactivation [7]. The high prevalence of $\mathrm{PsO}$ and hepatitis comorbidity and increasing demand for better therapeutic effects have made clinicians pay more attention to the issue of using biological agents reasonably in this at-risk population.

The current studies on this issue contained a small number of cases, and a targeted summary is necessary. Hence, in this systematic review and meta-analysis, we pooled the rates of hepatitis $B$ virus (HBV) reactivation among patients with $\mathrm{PsO}$ and $\mathrm{HB}$ treated with biological agents from several observational studies.

\section{METHOD}

The meta-analysis was conducted following the recommended items of Systematic Reviews and Meta-Analysis (PRISMA) guidelines [8] and Meta-analysis of Observational Studies in Epidemiology (MOOSE) guidelines [9]. This study is registered in PROSPERO (CRD42021243812, https://www.crd.york.ac.uk/prospero/\#record Details). This article is based on previously conducted studies and does not contain any new studies with human participants or animals performed by any of the authors.

\section{Literature Search}

Pubmed, Scopus, Embase, Cochrane Library, and Web of Science have been systemically searched to identify related studies before 
September 2021. Our retrieval strategies included three aspects: defined populations [patients with psoriasis/psoriatic arthritis (PsA) combined with hepatitis B], interventions (biological agents/treatments), and outcomes (reactivation of HBV). (Electronic Supplementary Material 1 shows the detailed search strategies.) All the reference lists of published reviews and journal articles have also been screened independently. We also searched OpenGrey (http://www. opengrey.eu) for gray Literature.

\section{Selection Criteria}

We included pieces of observational study that provided the original data on the HBV reactivation of the patients with $\mathrm{PsO}$ and $\mathrm{HB}$ after biological treatments. All the studies identified in the meta-analysis meet the following criteria: (1) definitive diagnosis of PsO/PsA with HB; (2) the reactivation rate was given or could be calculated. The number of cases presenting with HBV reactivation $(X) /$ the total number of patients included $(N), X / N$; (3) size of sample at least 10; (4) English journal articles. Literature that did not meet these criteria were excluded, as were (1) case reports, (2) conference abstracts, (3) reviews, (4) non-peer-reviewed materials, (5) patients diagnosed with other diseases and those treated with other immunosuppressive agents or immunomodulators during the treatment. Any disagreements were settled by discussion.

\section{Data Extraction and Statistical Analysis}

Two reviewers extracted and coded the data using an Excel spreadsheet independently. The data collected included the first author, year of publication, study design, sample size, age/sex distribution, the mean duration of biological treatment, serological detection of HBV markers, and types of biological agents. They verified each other's results to ensure the quality of data collection. Any differences were resolved through joint discussions with a third researcher to avoid potential bias.

\section{Quality Assessment}

The risk of methodological bias was evaluated on the basis of the modified Newcastle-Ottawa scale [10] from 11 domains as follows: sources of data, inclusion criteria, study period, the bias of evaluators, population-based or consecutive sampling, revalidation of outcomes, the control of confounding factors, exclusion criteria, processing of lost value, the integrity of the data collection, and follow-up assessment. Studies scoring at least eight points were regarded as high quality, while those scoring three points or less were of low quality.

\section{Statistical Analysis}

All analyses were conducted by Stata software (version 16.0). We pooled reactivation rates using random-effects models because of possible heterogeneity between studies. The Freeman-Tukey double arcsine transformation method was used in the process of calculation. Considering that the $Q$ statistic might be affected by the number of studies included, $I^{2}$ statistics and $H$ statistics were used to assess the statistical heterogeneity across the identified studies. After estimating the expected frequency, we chose the Fisher's exact test to compare multiple rates [11]. In addition, considering the sensitivity of the method and the number of studies included, we decided to use Egger's test to evaluate the potential publication bias. To find the sources of heterogeneity, subgroup analysis, influence analysis, and metaregression were performed. The predefined influencing factors included $\mathrm{HB}$ statuses, the types of biological agents (TNF $\alpha$ [tumor necrosis factor- $\alpha$ ] antagonists vs. non-TNF $\alpha$ inhibitors, namely indirectly antagonists of TNF $\alpha$ [interleukin-12/23 monoclonal antibodies or interleukin-17 inhibitors]), the duration of biological treatment (short-term/1 year or less vs. longterm/more than 1 year), the status of antiviral prophylaxis (no vs. yes). On the basis of the latest consensus [12] and available data, we classified $\mathrm{HB}$ status into three groups: (1) HBsAg-positive group, independently from the statuses of $\mathrm{HBcAb}, \mathrm{HBeAg}$, and $\mathrm{HBeAb}$ 
(including inactive carriers [serum HBV DNA $<2000 \mathrm{IU} / \mathrm{mL}$ ] and patients with chronic active hepatitis B [serum HBV DNA > $2000 \mathrm{IU} /$ $\mathrm{mL}]$ ). (2) HBsAg-negative, HBsAb-positive, and $\mathrm{HBcAb}$-positive group, namely past HBV infection or resolved HB. (3) HBsAg-negative, HBsAbnegative, and HBcAb-positive group. The patients in this group could also be subdivided into occult HBV infection (OBI), characterized by a small amount of serum/liver HBV-DNA $(<200 \mathrm{IU} / \mathrm{mL})$ and isolated HBcAb-positive (undetectable HBV DNA).

\section{RESULTS}

\section{Characteristics of Included Studies}

The detailed process of literature identification is shown in the PRISMA flow diagram (Fig. 1). First, we separated 1195 articles by the search terms from these databases (Embase, 730; Pubmed, 83; Cochrane Library, 1; Scopus, 116; Web of Science, 265). Two further articles were

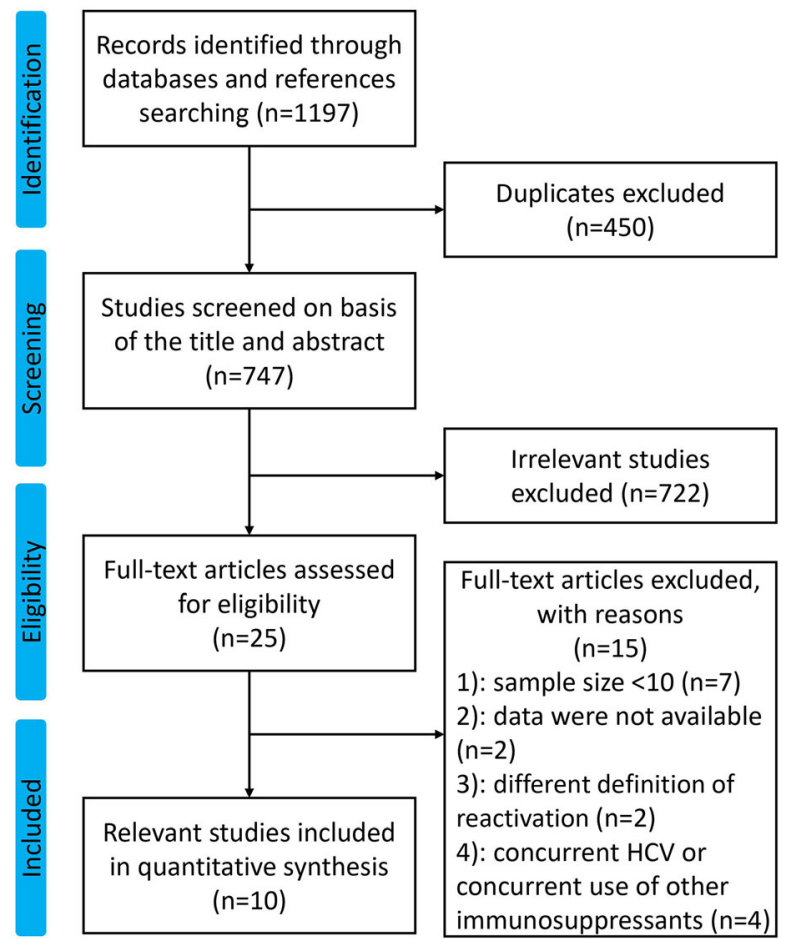

Fig. 1 PRISMA flow diagram for article screening and selection process found by reviewing the references and gray literature. After removal of 450 duplicates, 747 studies were left for screening titles and abstracts. Then 25 pieces of research needed a full-text review. Among them, seven were excluded because of the small sample size, six because HBV reactivation rates could not be extracted or calculated (mixed with data on other diseases or concurrent therapies), and two because of a different definition of HBV reactivation. Some studies containing cases that did not meet the inclusion criteria at the same time would be included after excluding these cases. Finally, we identified ten articles with a total of 238 subjects that met the eligibility criteria. The detailed characteristics of the identified ten studies are listed in Table 1.

\section{Risk of Bias of Included Studies}

We summarize the consequences of quality assessment in Fig. 2. All the studies were considered as having a moderate risk of bias.

\section{HBV Reactivation Rates}

HBV reactivation rates of different predefined groups are listed in Table 2.

\section{Overall Reactivation Rate}

As manifested in Fig. 3, the pooled HBV reactivation rate of total subjects was 0.018 (number $=10,95 \%$ CI $0.000-0.056$ ). There was no significant publication bias for included studies according to Egger's test result $(t=0.55$, $p=0.694$ ) and Funnel plot (see more details in Electronic Supplementary Material 2 and 3). The results of $I^{2}$ statistics $\left(I^{2}=38.04 \%\right.$, $p=0.104), \quad H$ statistics $\quad(H=1.27, \quad 95 \% \mathrm{CI}$ 1.00-1.87), and Galbraith plot showed no significant statistical heterogeneity across all the studies (see more details in Electronic Supplementary Material 4).

\section{Reactivation Rates of Different HB Statuses}

The reactivation rate of HBsAg-positive patients was $0.041 \quad(95 \% \mathrm{CI} \quad 0.000-0.179, \quad N=4$, $\left.I^{2}=0.00 \%, p=0.487\right)$. Of these, inactive carriers had a lower reactivation rate $(0.029,95 \% \mathrm{CI}$ 


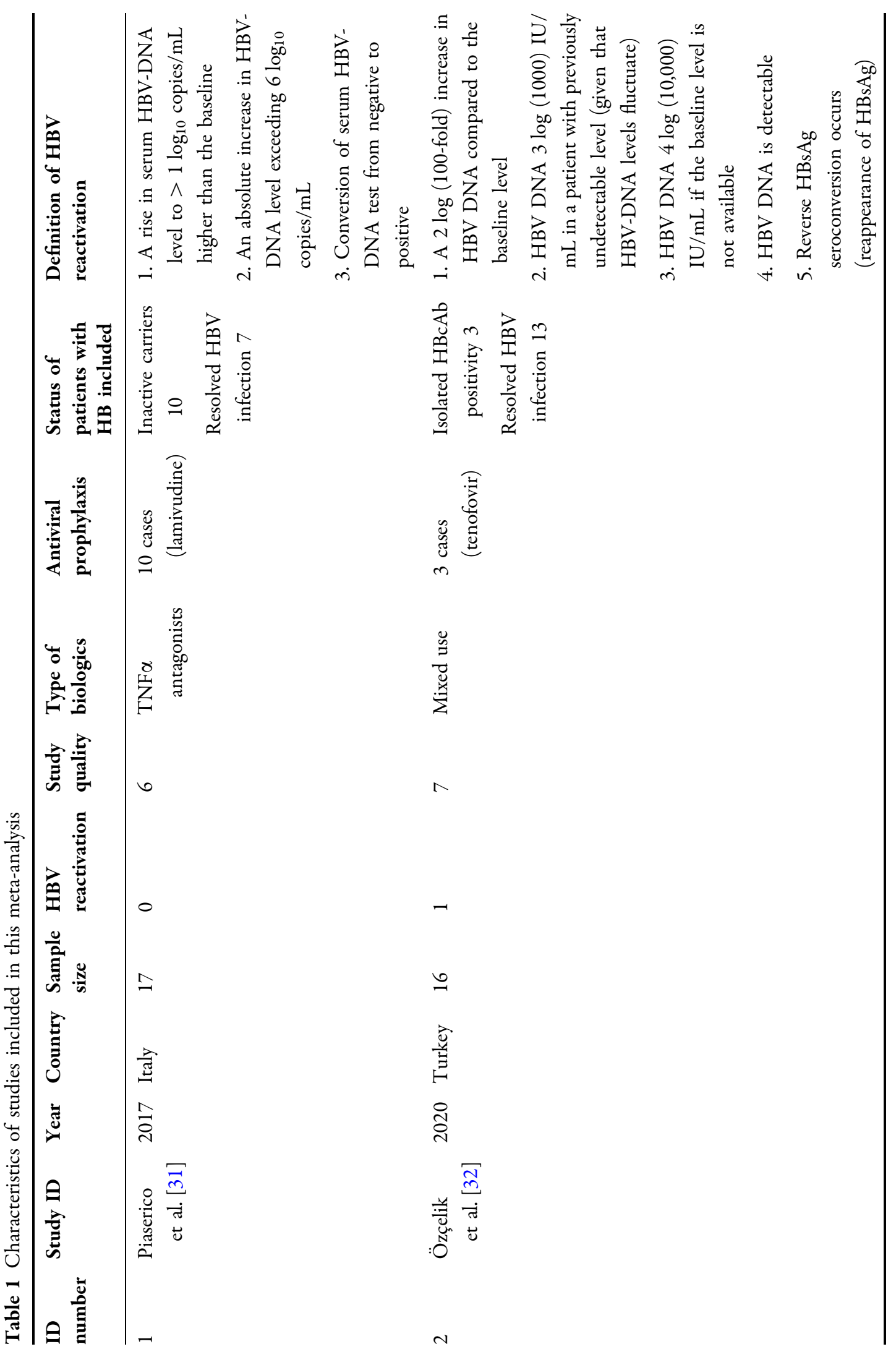




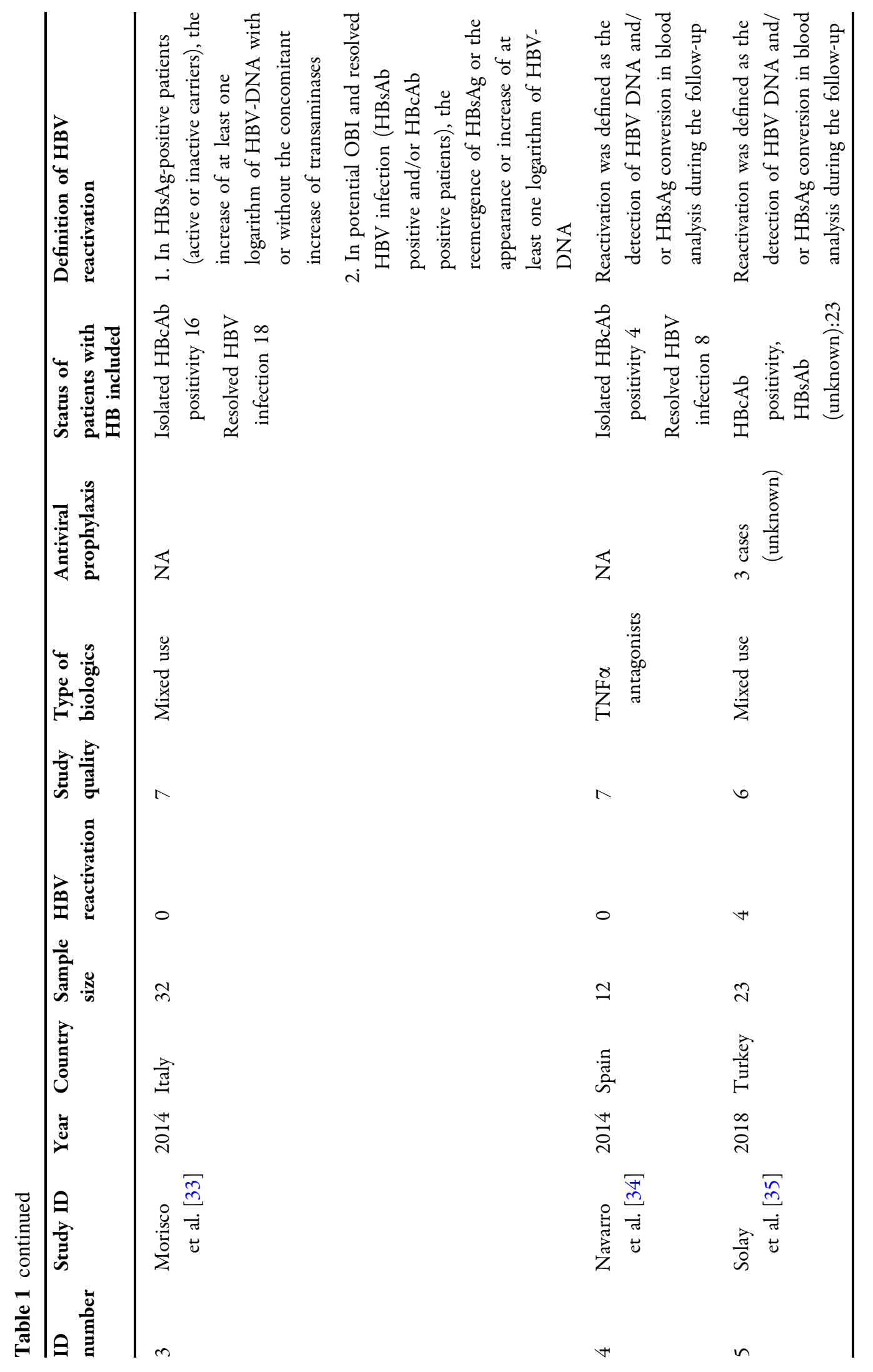




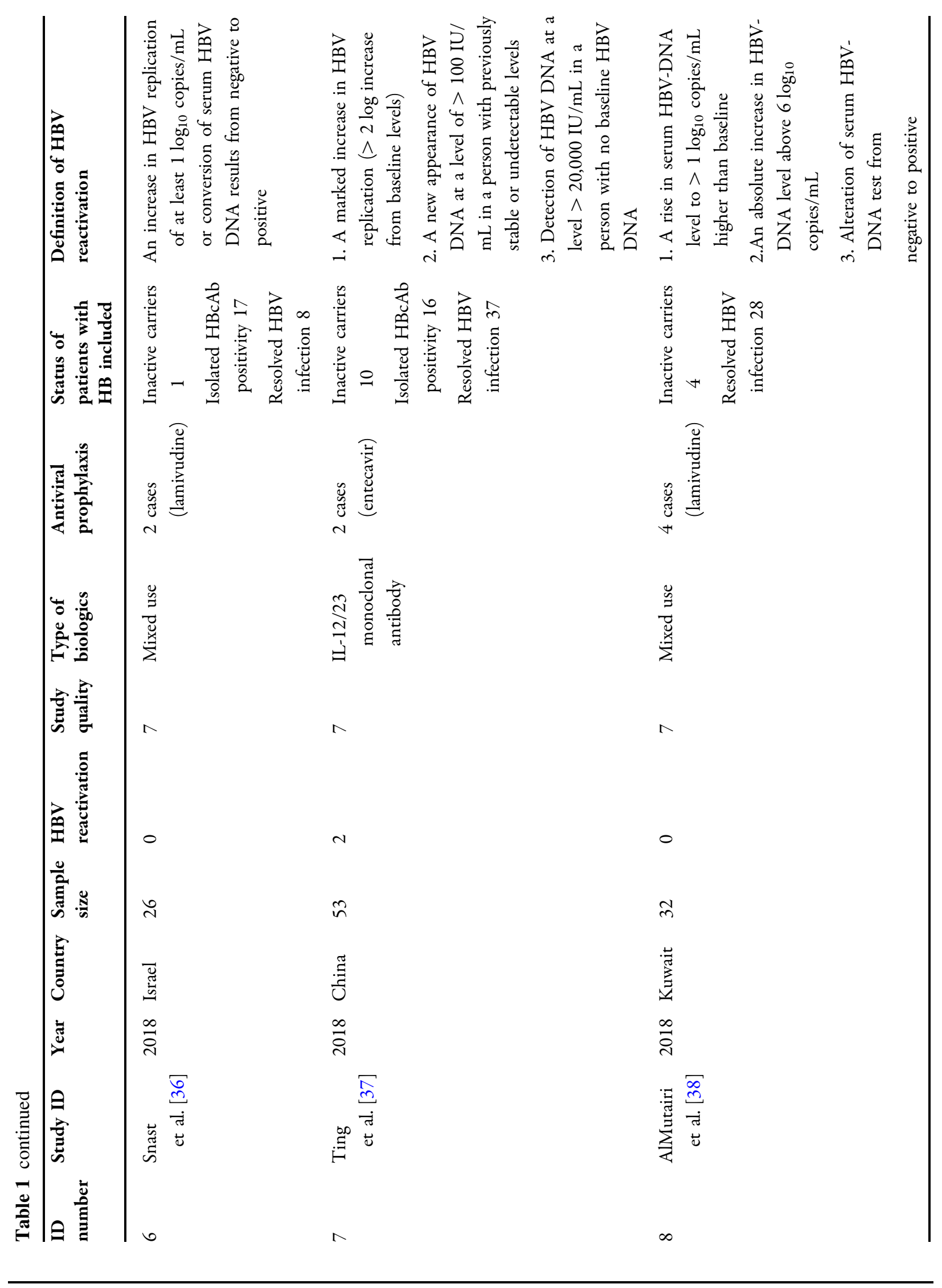




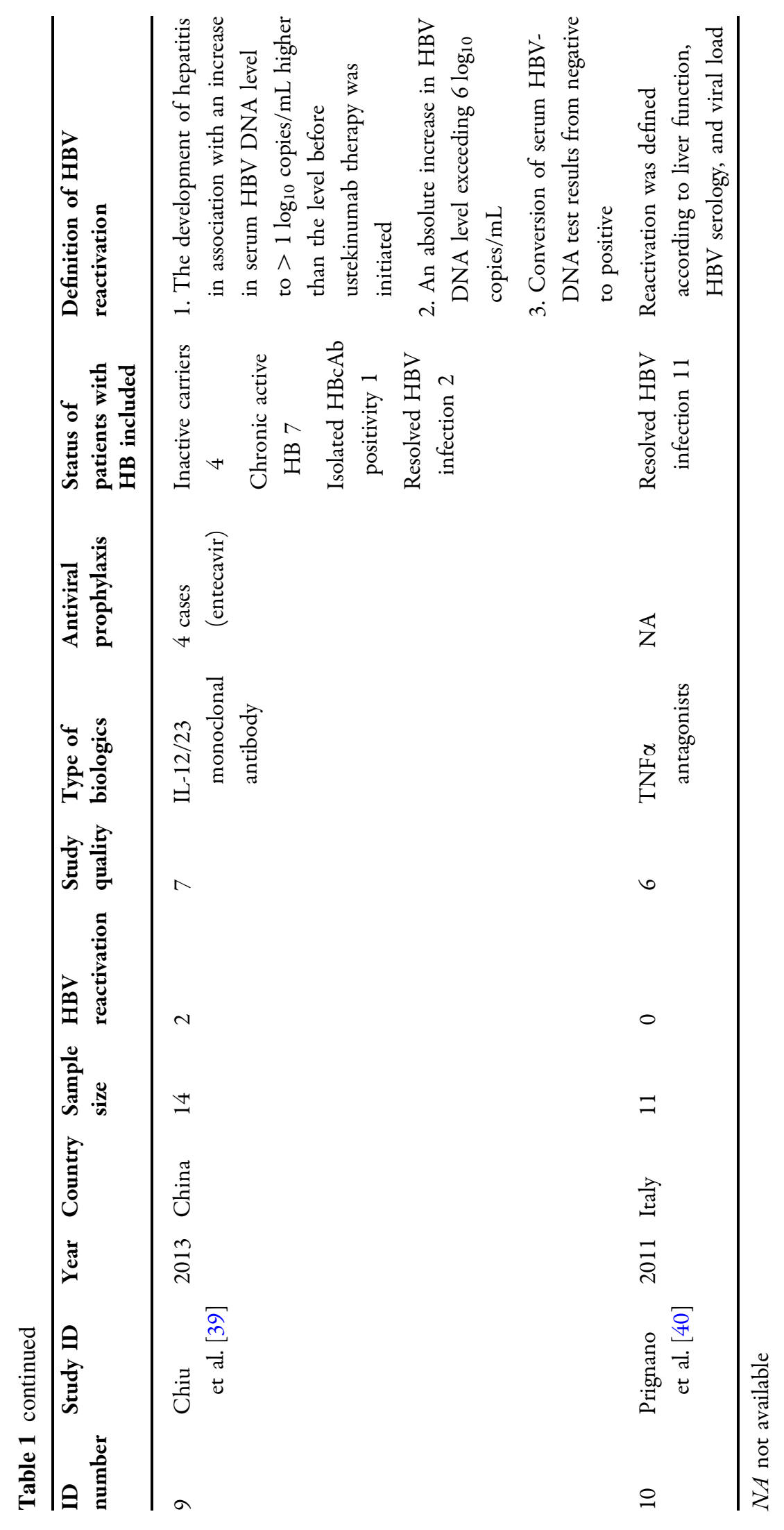




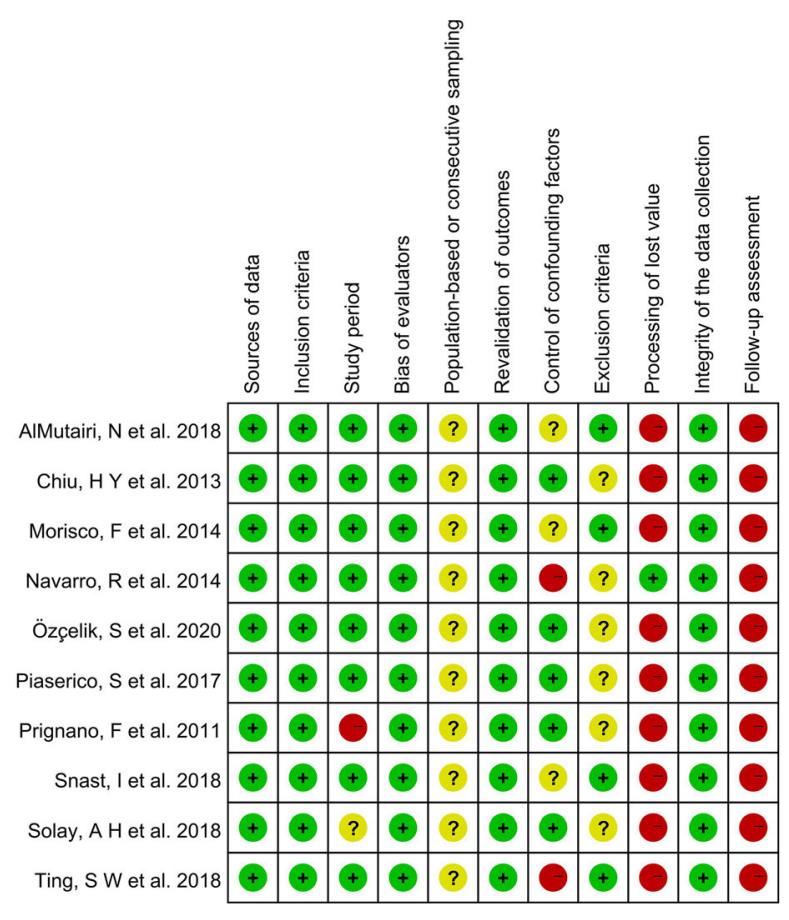

Fig. 2 Risk of bias summary

$\left.0.000-0.181, N=4, I^{2}=0.00 \%, p=0.451\right)$. The reactivation rate of all the HBsAg-negative patients was $0.002 \quad(95 \% \mathrm{CI} \quad 0.000-0.028$, $\left.N=10, I^{2}=25.55 \%, p=0.208\right)$. The results also showed that the rates of HBsAg-negative, $\mathrm{HBsAb}$-positive, and $\mathrm{HBcAb}$-positive group (past $\mathrm{HBV}$ infection or resolved $\mathrm{HB}$ ) and isolated $\mathrm{HBcAB}$-positive group (HBsAg-negative, HBsAbnegative without detectable $\mathrm{HBV}$ DNA) were 0.000 (95\% CI $0.000-0.020, N=8, I^{2}=0.00 \%$, $p=0.932)$ and 0.000 (95\% CI $0.000-0.008$, $\left.N=6, I^{2}=0.00 \%, p=0.979\right)$, respectively.

\section{Viral Reactivation Rates of Patients Using Different Types and Quantities of Biological Agents}

The HBV reactivation rate of the TNF $\alpha$ antagonists alone treatment group was 0.004 (95\% CI $0.000-0.055, N=5, I^{2}=0.00 \%, p=0.755$ ) while the rate of the non-TNF $\alpha$ inhibitors alone treatment group was $0.029 \quad(95 \% \mathrm{CI}$ $\left.0.000-0.098, N=3, I^{2}=0.00 \%, p=0.381\right)$. The group using only one kind of biological agent during one treatment episode had a rate of 0.014 (95\% CI 0.000-0.049, $N=8, I^{2}=0.00 \%$, $p=0.516)$. The viral reactivation value of the group using multiple types of biologics successively during the treatment was $0.000(95 \% \mathrm{CI}$ $\left.0.000-0.051, N=5, I^{2}=0.00 \%, p=0.681\right)$.

\section{Reactivation Rates of Different Statuses of Antiviral Prophylaxis}

The HBV reactivation rate was 0.266 (95\% CI $0.058-0.535, N=2$ ) in the patients who needed antiviral prophylaxis but did not receive it, and 0.000 (95\% CI $0.000-0.066, N=7, I^{2}=0.00 \%$, $p=0.999)$ in those who needed and accepted antiviral treatment. The patients with $\mathrm{HB}$ requiring antiviral therapy when treated with biologics are detailed in the "Discussion".

\section{Reactivation Rates of Different Durations of Treatment}

When the duration of biological treatment was 1 year or less, the viral reactivation rate of $\mathrm{HBV}$ was $0.057(95 \%$ CI $0.000-0.201, N=2)$. The corresponding value of the treatment group with a mean duration longer than 1 year was 0.005 (95\% CI $0.000-0.029, N=7, I^{2}=0.00 \%$, $p=0.700$ ). (See more details in Electronic Supplementary Material 5 Table S1 to Table S11 HBV reactivation rates of different predefined groups.)

\section{Statistical Analysis}

\section{Meta-Regression and Sensitivity Analysis}

Given the relatively small heterogeneity, we conducted meta-regression according to the predefined items and did not find the source of statistical heterogeneity. All the results are shown in Table 3. Influence analysis showed no significant difference between the total pooled rate and the combined reactivation rates after deleting any single study (HBV reactivation rates were all within the $95 \% \mathrm{CI}$ ). The result of sensitivity analysis confirmed that this outcome has good stability.

\section{Fisher's Exact Test}

This meta-analysis was a pairwise comparison between three groups, so we adjusted the inspection level $(0.05 / 3=0.0167)$. The results showed that the two-sided Fisher's exact test was 0.002 between HBsAg-positive and HBsAg- 
Table 2 Summary of hepatitis B virus reactivation rates

\begin{tabular}{|c|c|c|c|c|c|}
\hline Groups & $\begin{array}{l}\text { Number } \\
\text { of studies }\end{array}$ & $\begin{array}{l}\text { Heterogeneity } \\
(\%)\end{array}$ & $\begin{array}{l}\mathrm{HBV} \\
\text { reactivation }\end{array}$ & $95 \% \mathrm{CI}$ & $p$ value \\
\hline \multicolumn{6}{|l|}{ Hepatitis B status } \\
\hline $\begin{array}{l}\text { HBsAg-positive (inactive carriers or chronic active } \\
\text { hepatitis B) }\end{array}$ & 4 & $0.00 \%$ & 0.041 & $0.000-0.179$ & 0.487 \\
\hline $\begin{array}{l}\text { HBsAg-negative, } \mathrm{HB} \text { sb-positive, } \mathrm{HBcAb} \text {-positive } \\
\text { (resolved } \mathrm{HB} \text { or past } \mathrm{HBV} \text { infection) }\end{array}$ & 8 & $0.00 \%$ & 0.000 & $0.000-0.020$ & 0.932 \\
\hline $\begin{array}{l}\text { HBsAg-negative, } \mathrm{HBsAb} \text {-negative, } \mathrm{HBcAb} \text {-positive } \\
\text { (isolated } \mathrm{HBcAb} \text { positive or } \mathrm{OBI} \text { ) }\end{array}$ & 6 & $0.00 \%$ & 0.000 & $0.000-0.008$ & 0.979 \\
\hline \multicolumn{6}{|l|}{ Biological treatment } \\
\hline TNF $\alpha$ antagonists alone & 5 & $0.00 \%$ & 0.004 & $0.000-0.055$ & 0.755 \\
\hline Non-TNFa inhibitors alone & 3 & $0.00 \%$ & 0.029 & $0.000-0.098$ & 0.381 \\
\hline One kind of biologic during one treatment episode & 8 & $0.00 \%$ & 0.014 & $0.000-0.049$ & 0.516 \\
\hline Kinds of biologics during one treatment episode & 5 & $0.00 \%$ & 0.000 & $0.000-0.051$ & 0.681 \\
\hline \multicolumn{6}{|l|}{ Antiviral therapy } \\
\hline $\begin{array}{l}\text { Need antiviral therapy but did not receive } \\
\text { treatment }\end{array}$ & 2 & NA & 0.268 & $0.058-0.535$ & NA \\
\hline Need antiviral therapy and receive treatment & 7 & $0.00 \%$ & 0.000 & $0.000-0.066$ & 0.999 \\
\hline \multicolumn{6}{|l|}{ Duration of treatment } \\
\hline$\leq 1$ year & 2 & NA & 0.057 & $0.000-0.201$ & NA \\
\hline$>1$ year & 7 & $0.0 \%$ & 0.005 & $0.000-0.027$ & 0.700 \\
\hline
\end{tabular}

$O B I$ occult hepatitis $\mathrm{B}$ infection, $N A$ not available

aPatients with $\mathrm{HB}$ requiring antiviral therapy when treated with biologics are detailed in the "Discussion"

negative patients, and 0.007 among these three predefined HB status groups. Besides, the twosided Fisher's exact test between the inactive carriers and patients with chronic active hepatitis B was $1.000(p=0.923)$.

We also performed the same calculation between different categories and quantities of biological therapy groups. The outcomes of one-sided and two-sided Fisher's exact values between the two pre-classified types of biological agents were 0.227 and 0.371 , respectively. The values between the group using only one kind of biological agent and the other treatment group (at least two types) during one treatment episode were 0.663 and 1.000 . The results of two statuses of antiviral prophylaxis (need and accept it vs. need but not receive it) were calculated to be 0.023 . Besides, the exact values between different durations of biological therapy (1 year or less vs. more than 1 year) were all 0.104 .

\section{DISCUSSION}

This study quantified the risk of HBV reactivation in patients with $\mathrm{PsO} / \mathrm{PsA}$ and $\mathrm{HB}$ after biological treatment and compared the results 


\begin{tabular}{|c|c|c|c|c|c|c|}
\hline Study ID & Year & $N$ & $x$ & & $\operatorname{HBVr}(95 \% \mathrm{Cl})$ & Weight $\%$ \\
\hline Prignano, $\mathrm{F}$ et al. & 2011 & 11 & 0 & 1 & $0.000(0.000,0.285)$ & 6.29 \\
\hline Chiu, H Y et al. & 2013 & 14 & 2 & $\rightarrow$ & $0.143(0.018,0.428)$ & 7.48 \\
\hline Morisco, $\mathrm{F}$ et al. & 2014 & 34 & 0 & $\frac{1}{4}$ & $0.000(0.000,0.103)$ & 12.91 \\
\hline Navarro, $\mathrm{R}$ et al. & 2014 & 12 & 0 & -1 & $0.000(0.000,0.265)$ & 6.70 \\
\hline Piaserico, $\mathrm{S}$ et al. & 2017 & 17 & 0 & 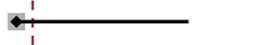 & $0.000(0.000,0.195)$ & 8.54 \\
\hline AlMutairi, $\mathrm{N}$ et al. & 2018 & 32 & 0 & $\frac{1}{1}$ & $0.000(0.000,0.109)$ & 12.51 \\
\hline Snast, I et al. & 2018 & 26 & 0 & 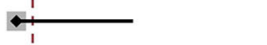 & $0.000(0.000,0.132)$ & 11.14 \\
\hline Solay, A H et al. & 2018 & 23 & 4 & $\longrightarrow$ & $0.174(0.050,0.388)$ & 10.36 \\
\hline Ting, S W et al. & 2018 & 53 & 2 & & $0.038(0.005,0.130)$ & 15.88 \\
\hline Özçelik, S et al. & 2020 & 16 & 1 & & $0.063(0.002,0.302)$ & 8.20 \\
\hline \multicolumn{4}{|c|}{ Overall $\left(I^{\wedge} 2=38.039 \%, p=0.105\right)$} & & $0.018(0.000,0.056)$ & 100.00 \\
\hline
\end{tabular}

Fig. 3 Pooled HBV reactivation rate. $X$ number of patients with hepatitis $B$ virus reactivation, $N$ number of patients enrolled in the research, $\mathrm{HBVr}$ hepatitis $\mathrm{B}$ virus reactivation

Table 3 Impact of study-level characteristics on effects of HBV reactivation after biological treatment, determined by metaregression

\begin{tabular}{lrcc}
\hline Characteristic & $\boldsymbol{t}$ value & 95\% CI & $\boldsymbol{p}$ value* $^{*}$ \\
\hline Degree of development & 0.26 & -0.824 to 0.973 & 0.810 \\
Study design & -0.20 & -1.234 to 1.087 & 0.852 \\
Proportion of HBsAg-positive patients & 0.06 & -0.638 to 0.661 & 0.959 \\
Duration of treatment & -0.26 & -0.758 to 0.642 & 0.809 \\
Proportion of antiviral prophylaxis $^{\mathrm{a}}$ & -0.07 & -1.143 to 1.091 & 0.945 \\
\hline
\end{tabular}

${ }^{*} p<0.05$

${ }^{2}$ Proportion of patients who needed and received antiviral prophylaxis

under different conditions. According to the risk categories, the overall risk of HBV reactivation (pooled rate $1.8 \%$ ) was relatively low to moderate [13]. All confirmation of viral reactivation cases was based on the changes in viral load, and none were due to the reverse conversion of serological markers (HBsAg).
First, we found that the differences in reactivation rate between different $\mathrm{HB}$ statuses were statistically significant after regulating the $p$ value $\left(p^{\prime}=0.0167\right)$. The HBV reactivation rate in the HBsAg-positive patients (4.1\%) was much higher, consistent with the consequences of a previous study [14] that HBsAg-positive patients were more likely to get $\mathrm{HBV}$ reactivated than 
their HBsAg-negative counterparts. In HBsAgpositive patients, the viral load in the serum would not significantly influence the reactivation rates of individuals with chronic active hepatitis B (one viral reactivation case in seven patients) and inactive carriers (2.9\%). Although the result of a meta-analysis on the role of surface antibody in hepatitis B reactivation showed that the risk of reactivation seemed to be significantly reduced in HBsAg-negative patients [15], the possibility still could not be completely ruled out. It was advisable to regularly use the triple serology (HBsAg, HBsAb, and HBcAb) to screen for HBV among these populations every 3-6 months [16]. HBsAb-positive status was deemed a protective factor against the reactivation of $\mathrm{HBV}$ in some studies. However, our result did not fully support this view since no significant statistical differences in viral reactivation rates between isolated HBcAb-positive patients and resolved patients with $\mathrm{HB}$ were found.

Second, our results also showed that the population that used $\mathrm{TNF} \alpha$ antagonists alone had a reactivation rate of $0.4 \%$, while the group that only used non-TNF $\alpha$ inhibitors has a rate of $2.9 \%$. Both kinds of treatment choices appeared to be safe statistically. Nevertheless, we could not conclude which kind of biological agent was much safer in treating patients with psoriasis and HB since the populations included in the two treatment groups (proportion of patients who received antiviral therapy, the status of $\mathrm{HB}$, age, and gender composition) mentioned above were not comparable enough. Some studies [17] considered that using TNFa antagonists in patients with psoriasis and $\mathrm{HB}$ was safer, while others [18] did not. Studies supporting the latter viewpoint had shown that $\mathrm{TNF} \alpha$ played a pivotal role in clearing and controlling HBV by suppressing viral replication [19]. The neutralization of TNF $\alpha$ would impair the process and facilitate chronic infections. Besides, the pooled reactivation rate of those treated with a single kind of biological agent (1.4\%) did not differ from the group using multiple biologics $(0.0 \%)$ during the treatment episode. Few patients would use different kinds of biologics simultaneously because the effectiveness and safety of the combined use were still unknown [20]. Successive use of multiple biologics had little effect on reactivation rate, probably owing to enough interval time (washout period) and no superimposed inhibition effects on pro-inflammatory cytokines.

The differences in treatment standards, medical insurance policies, and economic conditions in different countries and regions and no uniform guidelines available for clinicians to follow might be the reasons that led to different options and starting times for antiviral prophylaxis in patients with HB. Thus, after carefully reviewing many studies [21-23], we summarized that antiviral prophylaxis was required before biological therapies in the following patients: (1) HBsAg-positive patients (especially HBeAg-positive, HBV DNA copies $>2000 \mathrm{IU} / \mathrm{ml}$ patients), (2) Patients who need anti-CD20 monoclonal antibodies treatment or hematopoietic stem cell transplant. According to this, the highest reactivation rate (26.6\%) was found in individuals who needed antiviral precautions but did not accept them. The lack of antiviral treatment in these individuals would increase the risk of viral reactivation. The viral load of all the reactivation cases in our study returned to undetectable status 3-6 months after antiviral treatment began. One study [24] found that prompt initiation of antiviral therapy when reactivation occurs could prevent the development of symptomatic HB. Therefore, as some guidelines $[25,26]$ recommended, antiviral therapy should commence concomitantly or 1-2 weeks before biological treatments and be maintained for 6-12 months after terminating since reactivation typically occurred during the immune reconstitution. However, it did not mean that the use of antiviral treatment is a foolproof solution. The appearance of resistant mutant strains insensitive to anti-HBV medicines would lead to reactivation easily [27]. Therefore, regular follow-up and timely adjustment were advised during and after the antiviral therapies.

Another outcome of our concern was the duration of biological therapies. Among them, nine pieces of literature provided specific data. The duration of treatment ranged from 0.76 to 5.31 years. On the basis of the data available, we found that almost all reactivation cases 
occurred within 3 months to 1 year after the administration of biologics. Therefore, we took 1 year as the cutoff point. We found that prolonging the duration of biological treatment would not increase the risk of reactivation when HBV serological markers were well monitored. Most reactivation cases were more closely related to the progress of chronic $\mathrm{HB}$ and liver function than to the duration of treatment.

In addition to the quantifiable parameters above, we summarize other information of concern as follows. A multicentric prospective cohort study [28] using different doses of secukinumab (36 patients, $300 \mathrm{mg}$ vs. 13 patients, $150 \mathrm{mg}$ ) in patients with $\mathrm{PsO}$ and concurrent hepatitis B respectively found five and one cases of HBV reactivation after treatment, which meant that the changes of dosage of biological agents might affect the viral replication and reactivation.

Hepatitis has been defined in several studies as a fivefold or more increase in liver enzymes than normal. The extracted information showed that the liver enzymes in most cases did not change significantly during the treatment. Occasionally, liver enzymes were transiently elevated in very few cases, but none of them reached the diagnostic criteria for hepatitis, and most of these mild abnormalities in liver function would subside spontaneously. Among excluded studies, one [29] used the elevation of serum alanine aminotransferase (ALT) to define the reactivation of the HBV. However, previous studies had shown that the changes in serum HBV load were detected significantly days or weeks before the abnormal liver function occurred. It might not be sufficient and timely to evaluate HBV reactivation upon liver function alone. Besides, none of these studies mentioned any adverse events related to the biological treatments, including cirrhosis, hepatic failures, or other decompensated liver diseases.

Three of these ten identified studies mentioned the concomitant use of immunosuppressants or immunomodulators. Most of the patients added other systematic medicine because of the poor efficacy of biologics alone and did not experience viral reactivation. Only one patient using ustekinumab experienced virologic reactivation while receiving concurrent methotrexate. This patient had mild hepatitis without seroconversion of HBsAg. The combined use of systemic agents with biologics was previously confirmed to be more effective in treating psoriasis in the general population by randomized controlled study [30]. Nevertheless, it was not enough to conclude that the combined use was entirely safe for patients with $\mathrm{PsO} / \mathrm{PsA}$ and $\mathrm{HB}$, which still needed conclusive data for verifying in the future.

Finally, we did subgroup analysis, meta-regression, and sensitivity analysis to ensure the credibility of the results. The degree of development, study design, types of biological agents, the proportion of HBsAg-positive patients, and other predefined moderators could not explain the non-significant statistical heterogeneity in our data analysis process.

Our study had the following limitations: (1) No uniform diagnostic criteria were available for $\mathrm{HBV}$ reactivation, so that the research might be biased in the inclusion or exclusion of the target population. Clinical heterogeneity probably would arise during this process. (2) We did not calculate the reactivation rate of each biological agent in detail because of partial missing values. The safety and availability of the same category of biological agents could be different because of different administration route, dosage form, and other factors. (3) The estimate of reactivation risk might greatly rely on the study's design, which still needs more controlled clinical trials to verify.

\section{CONCLUSIONS}

HBsAg-positive patients with psoriasis are at high risk of virus reactivation when treated with biological agents without antiviral prophylaxis. Early and sufficient antiviral prophylaxis will effectively reduce the risk of HBV reactivation and serious complications in HBsAg-positive patients. The use of multiple biologics during one treatment episode has little effect on the reactivation rate. Prolonging the duration of biological treatment will not increase the risk of reactivation. Although there is a moderate risk of reactivation, the serum $\mathrm{HBV}$ antigen and 
antibody, HBV DNA level, and liver function should be meticulously determined and monitored regularly before and during biotherapies as well. Biological agents should be used cautiously in this population only after all other treatments have been exhausted. In the future, more randomized controlled studies with large samples are needed for better directing the clinical practices.

\section{ACKNOWLEDGEMENTS}

Funding. No funding or sponsorship was received for this study or publication of this article. The Rapid Service Fee was funded by the authors.

Authorship. All named authors meet the International Committee of Medical Journal Editors (ICMJE) criteria for authorship for this article, take responsibility for the integrity of the work as a whole, and have given their approval for this version to be published.

Author Contributions. Xinyu Wang and Ming Zhang led the analysis and writing of this manuscript. Yu Chen and Yirong Liu contributed to the hepatitis B aspect of this study. Xiaojie Huang and Yanqing Gao designed the study. Xinyu Wang, Ming Zhang and Yan Yu identified the articles, and extracted data from the articles that met the inclusion criteria. Xinyu Wang performed the statistical analysis.

Disclosures. Xinyu Wang, Ming Zhang, Chen Yu, Yirong Liu, Yan Yu, Xiaojie Huang, and Yanqing Gao have nothing to disclose.

Compliance with Ethics Guidelines. This article is based on previously conducted studies and does not contain any new studies with human participants or animals performed by any of the authors.

Data Availability. All data generated or analyzed during this study are included in this published article/as supplementary information files.
Open Access. This article is licensed under a Creative Commons Attribution-NonCommercial 4.0 International License, which permits any non-commercial use, sharing, adaptation, distribution and reproduction in any medium or format, as long as you give appropriate credit to the original author(s) and the source, provide a link to the Creative Commons licence, and indicate if changes were made. The images or other third party material in this article are included in the article's Creative Commons licence, unless indicated otherwise in a credit line to the material. If material is not included in the article's Creative Commons licence and your intended use is not permitted by statutory regulation or exceeds the permitted use, you will need to obtain permission directly from the copyright holder. To view a copy of this licence, visit http://creativecommons.org/licenses/by$\mathrm{nc} / 4.0 /$.

\section{REFERENCES}

1. Sbidian E, Chaimani A, Afach S, et al. Systemic pharmacological treatments for chronic plaque psoriasis: a network meta-analysis. Cochrane Database Syst Rev. 2020;1(1):D11535.

2. Ungprasert P, Erwin PJ, Koster MJ. Indirect comparisons of the efficacy of biological agents in patients with active ankylosing spondylitis: a systematic review and meta-analysis. Clin Rheumatol. 2017;36(7):1569-77.

3. Olbjørn C, Rove JB, Jahnsen J. Combination of biological agents in moderate to severe pediatric inflammatory bowel disease: a case series and review of the literature. Paediatr Drugs. 2020;22(4): 409-16.

4. Law ST, Taylor PC. Role of biological agents in treatment of rheumatoid arthritis. Pharmacol Res. 2019;150:104497.

5. Kim HJ, Lebwohl MG. Biologics and psoriasis: the beat goes on. Dermatol Clin. 2019;37(1):29-36.

6. Rongioletti F, Burlando M, Parodi A. Adverse effects of biological agents in the treatment of psoriasis. Am J Clin Dermatol. 2010;11(Suppl 1):35-7.

7. Loomba R, Liang TJ. Hepatitis B reactivation associated with immune suppressive and biological modifier therapies: current concepts, management 
strategies, and future directions. Gastroenterology. 2017;152(6):1297-309.

8. Page MJ, McKenzie JE, Bossuyt PM, et al. The PRISMA 2020 statement: an updated guideline for reporting systematic reviews. J Clin Epidemiol. 2021; 134:178-89.

9. Stroup DF, Berlin JA, Morton SC, et al. Meta-analysis of observational studies in epidemiology: a proposal for reporting. Meta-analysis of Observational Studies in Epidemiology (MOOSE) group. JAMA. 2000;283(15):2008-12.

10. Norris JM, Simpson BS, Ball R, et al. A modified Newcastle-Ottawa Scale for assessment of study quality in genetic urological research. Eur Urol. 2021;79(3):325-6.

11. Hess AS, Hess JR. Understanding tests of the association of categorical variables: the Pearson chisquare test and Fisher's exact test. Transfusion. 2017;57(4):877-9.

12. Nguyen MH, Wong G, Gane E, Kao JH, Dusheiko G. Hepatitis B virus: advances in prevention, diagnosis, and therapy. Clin Microbiol Rev. 2020. https:// doi.org/10.1128/CMR.00046-19.

13. Perrillo RP, Gish R, Falck-Ytter YT. American Gastroenterological Association Institute technical review on prevention and treatment of hepatitis $B$ virus reactivation during immunosuppressive drug therapy. Gastroenterology. 2015;148(1):221-44.

14. Cassano N, Mastrandrea V, Principi M, et al. Antitumor necrosis factor treatment in occult hepatitis B virus infection: a retrospective analysis of 62 patients with psoriatic disease. J Biol Regul Homeost Agents. 2011;25(2):285-9.

15. Paul S, Dickstein A, Saxena A, et al. Role of surface antibody in hepatitis $B$ reactivation in patients with resolved infection and hematologic malignancy: a meta-analysis. Hepatology. 2017;66(2):379-88.

16. Motaparthi K, Stanisic V, Van Voorhees AS, Lebwohl MG, Hsu S. From the Medical Board of the National Psoriasis Foundation: recommendations for screening for hepatitis B infection prior to initiating anti-tumor necrosis factor-alfa inhibitors or other immunosuppressive agents in patients with psoriasis. J Am Acad Dermatol. 2014;70(1):178-86.

17. Liu Y, Yang G, Zhang J, et al. Anti-TNF- $\alpha$ monoclonal antibody reverses psoriasis through dual inhibition of inflammation and angiogenesis. INT Immunopharmacol. 2015;28(1):731-43.

18. Furue K, Ito T, Furue M. Differential efficacy of biologic treatments targeting the TNF- $\alpha /$ IL-23/IL-17 axis in psoriasis and psoriatic arthritis. Cytokine. 2018;111:182-8.

19. Domm S, Cinatl J, Mrowietz U. The impact of treatment with tumour necrosis factor-alpha antagonists on the course of chronic viral infections: a review of the literature. Br J Dermatol. 2008;159(6):1217-28.

20. Korman NJ. Management of psoriasis as a systemic disease: what is the evidence? $\mathrm{Br} \mathrm{J}$ Dermatol. 2020;182(4):840-8.

21. Sarmati L, Andreoni M, Antonelli G, et al. Recommendations for screening, monitoring, prevention, prophylaxis and therapy of hepatitis B virus reactivation in patients with haematologic malignancies and patients who underwent haematologic stem cell transplantation-a position paper. Clin Microbiol Infect. 2017;23(12):935-40.

22. Reddy KR, Beavers KL, Hammond SP, Lim JK, FalckYtter YT. American Gastroenterological Association Institute guideline on the prevention and treatment of hepatitis B virus reactivation during immunosuppressive drug therapy. Gastroenterology. 2015;148(1):215-9.

23. Ogawa E, Wei MT, Nguyen MH. Hepatitis B virus reactivation potentiated by biologics. Infect Dis Clin N Am. 2020;34(2):341-58.

24. Cho YT, Chen CH, Chiu HY, Tsai TF. Use of antitumor necrosis factor- $\alpha$ therapy in hepatitis $B$ virus carriers with psoriasis or psoriatic arthritis: a case series in Taiwan. J Dermatol. 2012;39(3):269-73.

25. Menter A, Strober BE, Kaplan DH, et al. Joint AADNPF guidelines of care for the management and treatment of psoriasis with biologics. J Am Acad Dermatol. 2019;80(4):1029-72.

26. Bonifati C, Lora V, Graceffa D, Nosotti L. Management of psoriasis patients with hepatitis B or hepatitis C virus infection. World J Gastroenterol. 2016;22(28):6444-55.

27. Lampertico P, Viganò M, Manenti E, Iavarone M, Lunghi G, Colombo M. Adefovir rapidly suppresses hepatitis B in HBeAg-negative patients developing genotypic resistance to lamivudine. Hepatology. 2005;42(6):1414-9.

28. Chiu HY, Hui RC, Huang YH, et al. Safety profile of secukinumab in treatment of patients with psoriasis and concurrent hepatitis B or C: a multicentric prospective cohort study. Acta Derm Venereol. 2018;98(9):829-34.

29. Chiu YM, Lai MS, Chan KA. Commensurate incidence and outcomes of liver enzyme elevation between anti-tumor necrosis factor users with or 
without prior hepatitis B virus infections. PLoS ONE. 2018;13(4): e196210.

30. Busard C, Zweegers J, Limpens J, Langendam M, Spuls PI. Combined use of systemic agents for psoriasis: a systematic review. JAMA Dermatol. 2014;150(11):1213-20.

31. Piaserico S, Dapavo P, Conti A, Gisondi P, Russo FP. Adalimumab is a safe option for psoriasis patients with concomitant hepatitis $\mathrm{B}$ or $\mathrm{C}$ infection: $\mathrm{A}$ multicentre cohort study of 37 patients and review of the literature. J Eur Acad Dermatol Venereol. 2017;31(11):1853-9.

32. Özçelik S, Kılıç FA. Hepatitis B virus reactivation in patients with psoriasis on biologic therapies: a retrospective study. Turk Dermatoloji Dergisi. 2020;14(3):65-70.

33. Morisco F, Guarino M, La Bella S, Di Costanzo L, Caporaso N, Ayala F, Balato N. Lack of evidence of viral reactivation in $\mathrm{HBsAg-negative} \mathrm{HBcAb}$-positive and $\mathrm{HCV}$ patients undergoing immunosuppressive therapy for psoriasis. BMC Gastroenterol. $2014 ; 14: 214$.

34. Navarro R, Concha-Garzón MJ, Castaño C, Casal C, Guiu A, Daudén E. Outcome of patients with serology suggestive of past hepatitis B virus infection during antitumor necrosis factor therapy for psoriasis. Int J Dermatol. 2014;53(7):909-11.
35. Solay AH, Acar A, Eser F, Kuşcu F, Tütüncü EE, Kul G, Şentürk GÇ, Gürbüz Y. Reactivation rates in patients using biological agents, with resolved $\mathrm{HBV}$ infection or isolated anti-HBc IgG positivity. Turk J Gastroenterol. 2018;29(5):561-5.

36. Snast I, Atzmony L, Braun M, Hodak E, Pavlovsky L. Risk for hepatitis $\mathrm{B}$ and $\mathrm{C}$ virus reactivation in patients with psoriasis on biologic therapies: A retrospective cohort study and systematic review of the literature. J Am Acad Dermatol. 2017;77(1): 88-97.

37. Ting SW, Chen YC, Huang YH. Risk of hepatitis B reactivation in patients with psoriasis on ustekinumab. Clin Drug Invest. 2018;38(9):873-80.

38. AlMutairi N, Abouzaid HA. Safety of biologic agents for psoriasis in patients with viral hepatitis. J Dermatol Treat. 2018;29(6):553-6.

39. Chiu HY, Chen CH, Wu MS, Cheng YP, Tsai TF. The safety profile of ustekinumab in the treatment of patients with psoriasis and concurrent hepatitis B or C. Br J Dermatol. 2013;169(6):1295-303.

40. Prignano F, Ricceri F, Pescitelli L, Zanieri F, Lotti T. Tumour necrosis factor- $\alpha$ antagonists in patients with concurrent psoriasis and hepatitis B or hepatitis C: A retrospective analysis of 17 patients. $\mathrm{Br} \mathrm{J}$ Dermatol. 2011;164(3):645-7. 